\begin{tabular}{|c|c|}
\hline \multirow{3}{*}{ 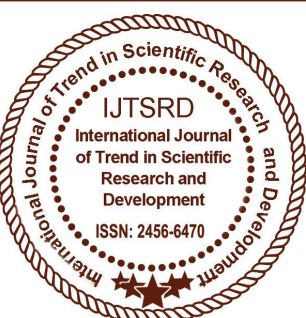 } & $\begin{array}{l}\text { International Journal of Trend in Scientific } \\
\text { Research and Development (IJTSRD) }\end{array}$ \\
\hline & International 0 \\
\hline & ISSN No: 2456 - 6470 | www.ijtsrd.com | Volume - 2 | Issue -4 \\
\hline
\end{tabular}

\title{
An Efficient FIR Filter for Electrocardiogram Signal
}

\author{
D. Kousaly. $A^{1}$, Dr. B. Senthilkumar ${ }^{2}$ \\ ${ }^{1}$ Research Scholar, ${ }^{2}$ Professor \\ Kalaignar Karunanidhi Institute of Technology, Coimbatore, Tamil Nadu, India
}

\section{ABSTRACT}

Filtering of Electrocardiogram signal is very important because noisy Electrocardiogram signal can mask some important features of the Electrocardiogram. Graphical information related to the heart functioning by means of a base line and waves representing the heart voltage changes during a period of time, usually a short period is given by Electrocardiogram. It is done by putting leads on specific part of the human body, to get changes of the bioelectrical heart signal. Electrocardiogram is considered to be a must have feature for a medical diagnostic imaging system. Electrocardiogram, a non invasive technique is used as a primary diagnostic tool for cardiovascular diseases. A cleaned Electrocardiogram signal provides necessary information about the electrophysiology of the heart diseases and ischemic changes that may occur. It provides valuable information about the functional aspects of the heart and cardiovascular system. The objective of the work is to automatic detection of cardiac arrhythmias in Electrocardiogram signal in Pan Tompkins tern reorganization technique is used in this project for detection of cardiac arrhythmias. The proposed system consists of three main stages, which are the Electrocardiogram. In the first stage, the Electrocardiogram various processing is responsible for three tasks: theye are 1) Electrocardiogram filtering; 2) QRS complex detection; and 3) $\mathrm{T}$ and P wave delineation. This system has memories, filter, PAT algorithm and naive base classifier using xlinx software tool and finally the normal or abnormal signal is determined by the normal or abnormal flags.
Keywords: Electrocardiogram (ECG), QRS complex detection, Electrocardiogram filtering, Pan and Tompkins (PAT) algorithm, naive base classifier

\section{INTRODUCTION}

The Electrocardiogram filtering removes the noise coupled with the Electrocardiogram signal and prepares it for further analysis. After that, the QRS complex is detected using the Pan and Tompkins algorithm. Finally $\mathrm{T}$ and $\mathrm{P}$ waves are delineated, and the corresponding fiducially points ( $\mathrm{P}$ onset, $\mathrm{P}$ peak, $\mathrm{P}$ offset, $\mathrm{T}$ onset, $\mathrm{T}$ peak, and $\mathrm{T}$ offset) are extracted. This system has memories, filter, PAN TOMPKINS algorithm and naive base classifier and finally the normal or abnormal signal is determined by the normal or abnormal flags.

The electrocardiography (ECG) is defined as the recording of the electrical activity of the heart [1]. These electrical signals are directly related to the normal mechanical functioning of the heart. Thus, Electrocardiogram is very commonly used as a preliminary non-invasive test to detect the normal functioning of heart. Filtering of Electrocardiogram signal after acquisition is necessary to remove noise, as noise may arise due to movement of subject, electronic noise of instrumentation amplifiers, power line and electromagnetic interference. Any signal other than that of interest can be termed as noise. The Electrocardiogram signal is a relatively strong signal with a readily identifiable waveform. The various noises and interferences, as mentioned earlier, affecting Electrocardiogram signals can be removed by band pass filters [2]. Since Electrocardiogram is a non-stationary signal, removal of noise is not an easy task. [3] Removal of noise from Electrocardiogram sometimes may lead to loss of information as noise 
and information lie in an overlapped range in the signal spectrum. Hence every denoising technique is aimed at minimal loss of information with satisfactory level of noise elimination [4]. Various filtering techniques and filter designs are available for Electrocardiogram filtering, thus choosing the right filter for the purpose is a problem. It was seen that Butterworth, Chebyshev, elliptic and Bessel filters are the most commonly used filters [2], but their performance need to be quantified and compared to successfully choose the right filter design. Another commonly used technique is Wavelet Denoising. It has been seen that performance of Wavelet based filters depends of the amount of noise present in the signal. When the amount of noise is low, IIR filters are better at removing noise compared to wavelet based filters, but when noise coverage is greater than $50 \%$, wavelet based filters showed better SNR. Also, computation times are shorter in case of IIR filters when compared to wavelet based filters [5]. Other methods have been recently used by some authors for Electrocardiogram signal denoising. Some of these methods are - Empirical Mode Decomposition (EMD), Independent Component Analysis (ICA), adaptive filtering [6] [7] [8]. In another study it has been observed that FIR and IIR filters show maximum SNR improvement when used to remove powerline interference. Hence these simple filters arequite commonly used for Electrocardiogram signal denoising [9]. This study thus compares the performance of various Finite Impulse Response (FIR) and Infinite Impulse Response (IIR) filters for Electrocardiogram signal noise removal based on their signal-to noise ratio (SNR), and thus aims to find the most suitable filter design and the optimum order at which it will effectively remove noise from Electrocardiogram. Performance analysis of the filters is based on SNR of the filters at various orders. SNR is defined as the dimensionless ratio of the signal power to the noise power [10]. Thus, SNR quantifies the amount of noise present in the signal with respect to the signal of interest. Thus, SNR has been used the parameter for comparison of various filter designs.

It concluded with the comment that wavelet transform (WT) method with average compression ratio of 86.41 and percentage root mean square of 0.346 provide better compression and fidelity than FFT and DCT compression algorithms. But wavelet transform (WT) is a computationally intensive process and very slow when computed by general purpose computing system [12]. To the author's knowledge, no real life implementation of such complex algorithm for Electrocardiogram data compression have reported. In recent, direct data compression based, DPCM with run length encoding (RLE) Electrocardiogram data compression method has been proposed in [13]. Method is found to be simple from computation and implementation on low end microcontroller point of view for real time Electrocardiogram monitoring. Proposed method achieved compression ratio 5.92 and PRD 8.19 using MITBIH databases [13]. Lossless Electrocardiogram data compression based on ASCII character encoding has been proposed in [14] and fidelity achieved is better. But it is found that use of magnitude encoding, run length encoding and ASCII character encoding for Electrocardiogram data compression with fix length of encoded character leads to poor bits per sample (BPS) efficiency in low slope and equipotential regions of Electrocardiogram [15]. Electrocardiogram data compression algorithm based on dynamic bit allocation strategy and delta encoding has been proposed in [15]. Use of dynamic bit allocation strategy has improved bits per sample (BPS) efficiency in real time Electrocardiogram data compression.

\section{LITERATURE SURVEY}

Base on Field Programmable Gate Array (FPGA), Electrocardiogram compression by using discrete cosine transform (DCT) has been proposed in [16]. For compression purpose ELECTROCARDIOGRAM signal represented into sets of 300 samples called frames with sampling frequency of $250 \mathrm{~Hz}$. Originally each frame required $300 \times 12=3600$ bits after FPGA based compression using DCT algorithm, it reduced to 960 bits i.e. compression ratio achieved 3.75 where as theoretical value is 7 and 4.23 PRD achieved with the implementation [16]. Improved linear approximation distance threshold (LADT) algorithm has been proposed in [17]. Xilinx XC35400 [17] FPGA has been used for implementation and VHDL as hardware description language. Compression ratio obtained is 6 [17]. RTL schematic for improved LADT based Electrocardiogram data compressor has also been illustrated in paper. For adaptive low-power design for biometrics and healthcare applications [18] primary aim was to implement wireless body sensor network (WBSN) [18] system with low power, cost and complexity requirement in order to monitor physiology signals such as body temperature, electrocardiogram (Electrocardiogram), pulse oxygen saturation, blood pressure, blood glucose etc. for 24- 
hour. FPGA used to emulate the function of MCU. For Electrocardiogram data compression CR achieved 1.92 , total power consumption $150 \mu \mathrm{W}$ and gate count 13.4K [18]. A low power Electrocardiogram signal processor (ESP) has been proposed in [19]. The final ESP chip photograph is presented in paper. Proposed ESP performs three different operations, first filter the Electrocardiogram signal then Electrocardiogram compression and finally encryption. Skeleton and Huffman coding [19] are employed to compress the Electrocardiogram signal. Compression ratio of 8.4 (Skeleton) / 2.1 (Huffman) are obtained and compression error i.e. PRD of 0.641 achieved [19]. At operating frequency of $1 \mathrm{KHz}$, power consumption of $6 \mu \mathrm{W}$ has been noted using $0.18 \mu \mathrm{m}$ technology. Single chip implementation for the DOT/EEG/Electrocardiogram multiprocessor with the lossless data compressor using $65 \mathrm{~nm}$ CMOS technology has been proposed in [20]. Hardware architecture for the mixed bio-signal lossless compression is provided in paper. Primary aim was to provide the reduce Electrocardiogram storage and communication bandwidth requirement using VLSI implementation of mixed bio-signal with lossless data compressor. DPCM prediction followed by GolombRice entropy coding implemented on chip and compression ratio of 2.38 achieved with lossless compression [20]. With $0.065 \mu \mathrm{m}$ technology and $24 \mathrm{MHz}$ operating frequency, $170 \mu \mathrm{W}$ power consumption observed for chip [20]. In recent, Electrocardiogram lossless encoder for wireless healthcare monitoring application has been proposed in [21]. Adaptive trending prediction and a two-stage entropy coding algorithm [21] based lossless Electrocardiogram encoder architecture is proposed in paper. This implementation is based on Huffman coding since it gives variable-length codes (VLCs) for source fixed-length codes (FLCs) [21]. With $0.18 \mu \mathrm{m}$ technology implementation, compression ratio of 2.43 achieved [21]. Power consumption of $36.4 \mu \mathrm{W}$ observed at operating frequency of $100 \mathrm{MHz}$ [21]. Lossless Electrocardiogram compression with fuzzy decision has been proposed in [22]. Fuzzy decision controller architecture for adaptive prediction has been proposed. Compression ratio of 2.53 achieved with two stage Huffman encoder as entropy encoder. Chip layout is given with $0.18 \mu \mathrm{m}$ technology implementation. At the operating frequency of $100 \mathrm{MHz}$, power consumption of $28.3 \mu \mathrm{W}$ is noted. In [23], system-onchip (SoC) implementation of Electrocardiogram compressor is proposed. System architecture of proposed SoC has been given in paper also using linear slope predictor and dynamic codingpackaging scheme [23] to frame the resulting error compression algorithm has been proposed. Total power consumption by Electrocardiogram-SoC is $535 \mathrm{nW}$ [23]. With proposed SoC using $0.35 \mu \mathrm{m}$ technology and total gate count of $2.26 \mathrm{~K}$, average $2.25 \mathrm{x}$ compression ratio is achieved [23]. Adaptive region prediction based lossless Electrocardiogram compression implementation has been proposed in [24]. To make simpler transmit format of different Electrocardiogram signal region, variable length coding is used to encode prediction difference. Implementation using $0.18 \mu \mathrm{m}$, compression ratio of 2.67 is achieved with power consumption of $127 \mu \mathrm{W}$ [24]. Hardware architecture has also been given in paper for the adaptive region prediction based Electrocardiogram compression. Low complexity and high performance lossless ELECTROCARDIOGRAM data compression using fuzzy decision and particle swarm optimizer (PSO) [25] has been proposed in [25]. Using proposed fuzzy-based PSO prediction and Huffman region entropy-coding techniques, average compression ratio of 2.84 is achieved for different MIT-BIH Arrhythmia database. Power consumption by VLSI architecture is $201 \mu \mathrm{W} /$ with $0.09 \mu \mathrm{m}$ technology and operating frequency of $200 \mathrm{MHz}$ [25]. Layout photograph and chip specifications are given in paper. Above the existing system analysis need employing the efficient fir filter for electrocardiogram signal.

\section{SYSTEM DESIGN}

In the proposed system, the process of Electrocardiogram signal extraction is discussed by replacing the filter with and without pipelined structure. An Efficient FIR filter with modified booth multiplier structure is designed with and without pipelining. The designed filter is replaced in the Electrocardiogram signal processor and the reduction in the delay and high speed is achieved. This system has memories, filter, PAN TOMPKINS algorithm and naive base classifier and finally the normal or abnormal signal is determined by the normal or abnormal flags. 


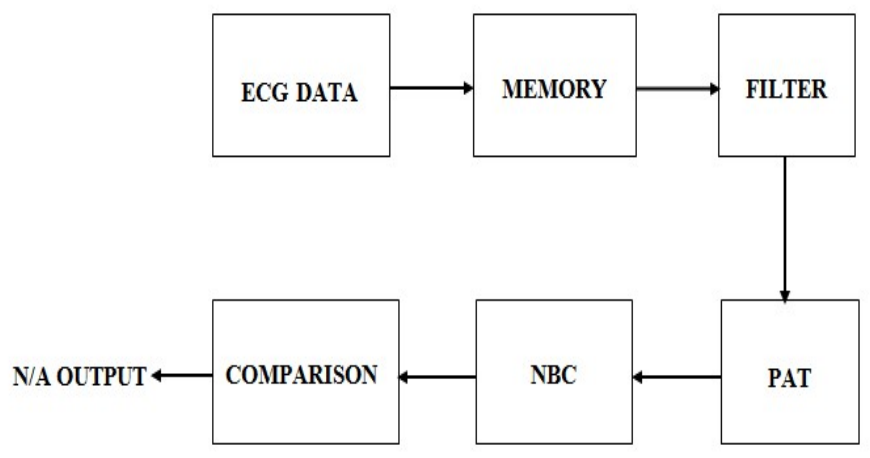

Fig 1 Block Diagram of Digital ELECTROCARDIOGRAM

The proposed system is a life saviour for Pan Tompkins algorithm who are susceptible to ventricular arrhythmia by alerting them for immediate attention to their medical condition. Unlike other systems that acquire the Electrocardiogram signal and transmit it for further analysis, the proposed system aims to design and develop an integrated biomedical processor that is capable of acquiring the Electrocardiogram signal from the heart along with processing and analysing it on the same chip without any external interaction. Thus, the Pan Tompkins algorithm would have immediate alert to his situation and that is very important, especially in critical situations.

\section{A. FIR FILTERS}

Digital filters can be divided into two categories: finite impulse response (FIR) filters; and infinite impulse response (IIR) filters. Although FIR filters, in general, require higher taps than IIR filters to obtain similar frequency characteristics, FIR filters are widely used because they have linear phase characteristics, guarantee stability and are easy to implement with multipliers, adders and delay elements. The number of taps in digital filters varies according to applications. In commercial filter chips with the fixed number of taps, zero coefficients are loaded to registers for unused taps and unnecessary calculations have to be performed. To alleviate this problem, the FIR filter chips providing variable-length taps have been widely used in many application fields. However, these FIR filter chips use memory, an address generation unit, and a module unit to access memory in a circular manner. The paper proposes two special features called a data reuse structure and a recurrent-coefficient scheme to provide variable-length taps efficiently. Since the proposed architecture only requires several MUXs, registers, and a 39 feedback-loop, the number of gates can be reduced over $20 \%$ than existing chips.

\section{B. PAN TOMPKINS ALGORITHM:}

Pan Tompkins is one of the methods for the detection of Electrocardiogram waves. The QRS detection algorithm introduced by Pan and Tompkins is the most widely used and often cited algorithm for the extraction of QRS complexes from electrocardiograms. The methodology followed is that the Electrocardiogram is passed through a low-pass and a high-pass filter in order to remove noise from the signal. Then the filtered signal is passed through derivative, squaring and window integration phases. Finally, a thresholding technique is applied and the Rpeaks are detected

\section{NBC}

Classification is a form of data analysis that extracts models describing important data classes. Classification has numerous applications, including fraud detection, target marketing, performance prediction, manufacturing, and medical diagnosis because of high accuracy, prediction rate and automatic method to search

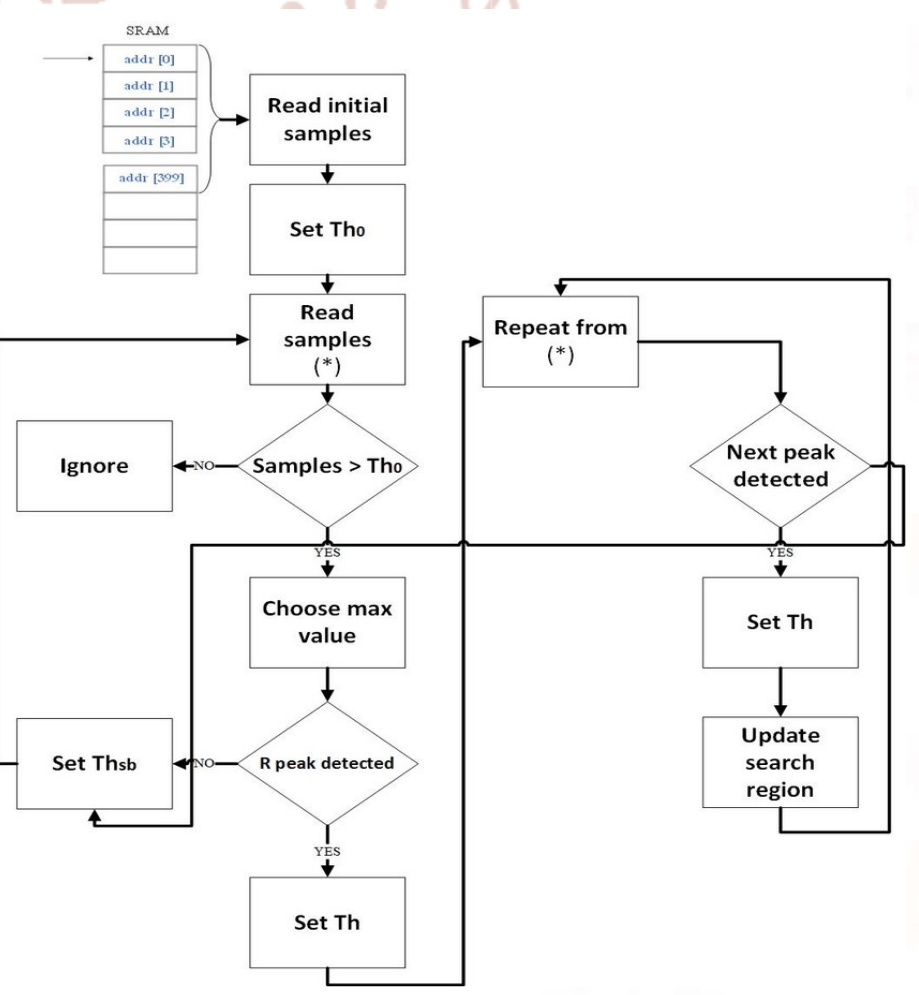

Fig 2 Flowchart of PAN TOMPKINS Algorithm

For hypothesis. Algorithms like SVM, NB, decision trees, hiking etc are used for diverse sorts of classification and prediction. In this phase, the Naïve Bayes (NB) classifier is used to classify the Electrocardiogram signal as Normal and Abnormal implemented in Rapid Miner tool. NB classifiers are statistical classifiers can predict class membership probabilities such as the probability that a given tuple 
belongs to a particular class. Bayes classifiers have also exhibited high accuracy and speed when applied to large databases.

\section{RESULTS AND DISCUSSION}

In order to detect the abnormality in Electrocardiogram, pre-processing stage is followed by R-peak detection and beat classification. The simulation experiments are based on manual database Electrocardiogram signal for performing the simulation study, because of its wide acceptability. The real time recorded data using National Instruments (NI) DAQ also utilized for this study. The data base consists of 30-min excerpts of two-channel ambulatory Electrocardiogram recordings. The database Electrocardiogram signal is originally obtained by placing the electrodes on the chest in the first channel V1that is the standard practice in Electrocardiogram recording. The recorded signal has been digitized at $540 \mathrm{~Hz}$ sampling rate with 11-bit resolution over a $10 \mathrm{mV}$ range. All the simulation experiments were conducted using Xilinx .In the simulation scenario; we choose $\mu$ for all the filters as 0.01 and the filter length as 21 for reducing the simulation time. The number of iterations for the experiment. kit is used for extracting frequency domain features such as low frequency, high frequency, frequency ratio and normalized frequency. Filter convergence vary in accordance to step size, it converges faster for high step size. MSE reduces in accordance with the increase in SNR of noise. MSE of $19.5 \mathrm{~dB}$ has been achieved for the iteration time110 in PAN TOMPKINS algorithm which is superior to other, transform domain (TDLMS) and delayed NLMS (DNLMS) algorithms. the waveforms of the simulation for various inputs and output parameter using the Xilinx tool. The pre-processed Electrocardiogram is applied to R-peak detection stage to determine the possible beats per minute. The human heart rate values vary between 60 to $100 \mathrm{bpm}$. Manually pre defined set point is used and detect the $\mathrm{RR}$ interval as well as possible R-peaks and beat rate obtained is 72. The coefficients are calculated and plotted for real time recorded signals and database in figure 4 .
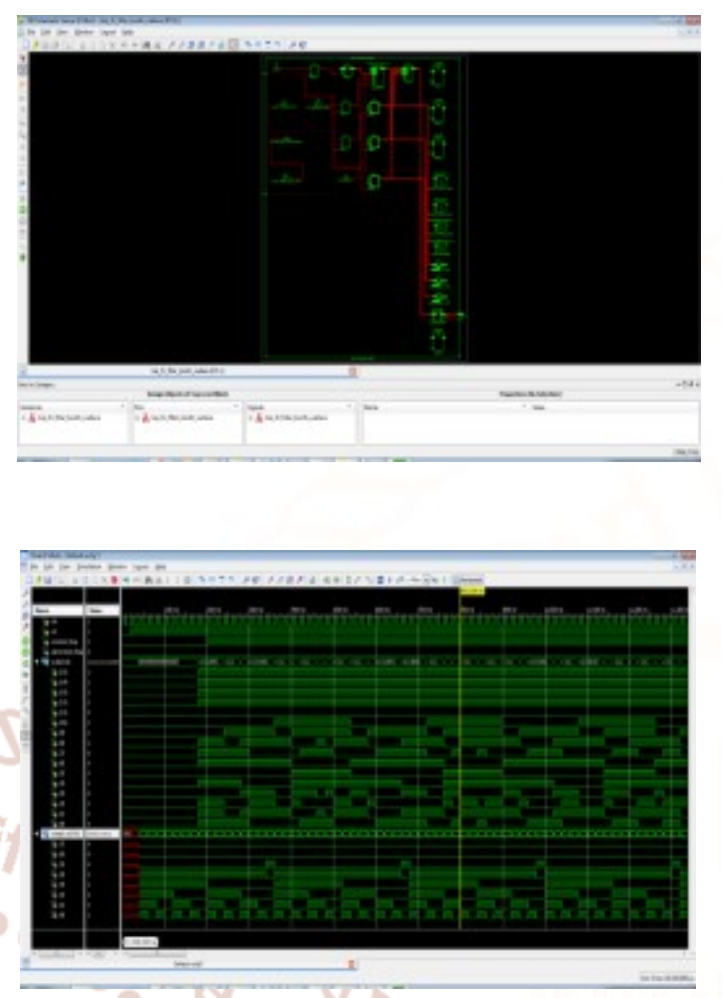

Fig 3 RTL Structure Simulation Result of ELECTROCARDIOGRAM Signal with Non Pipelined Structure

\begin{tabular}{|l|c|c|}
\hline PARAMETER & $\begin{array}{c}\text { EXISTING - } \\
\text { BOOTH } \\
\text { MULTIPLIER } \\
\text { WITH } \\
\text { WALLACE } \\
\text { TREE }\end{array}$ & $\begin{array}{c}\text { PIPELINED - } \\
\text { BOOTH } \\
\text { MULTIPLIER } \\
\text { WALLACE } \\
\text { TREE }\end{array}$ \\
\hline Slice LUTs & 79 & 78 \\
\hline Slice Flip Flops & - & 52 \\
\hline Delay & $13.503 \mathrm{~ns}$ & $5.122 \mathrm{~ns}$ \\
\hline Frequency & - & $195.255 \mathrm{MHz}$ \\
\hline
\end{tabular}

Table 1 Comparison of the Multiplier with and Without Pipelining

Above the table 1 proposed system compared with existing one delay is better than existing system. Existing system frequency 13.50 but proposed system frequency nearly half of the existing system (5.122).also proposed system other parameters above the table better than existing system The Efficient FIR filter is designed with and without pipelining and the delay in the filter is compared. On comparing the delay the pipelined structure gives very less delay while compared with the non pipelined structure and the frequency in the pipelined structure is higher than the non pipelined structure which shows the increase in the speed. 


\begin{tabular}{|l|c|c|}
\hline PARAMETER & $\begin{array}{c}\text { FIR - BOOTH } \\
\text { MULTIPLIER } \\
\text { WITH } \\
\text { WALLACE } \\
\text { TREE }\end{array}$ & $\begin{array}{c}\text { FIR - } \\
\text { PIPLINED - } \\
\text { BOOTH } \\
\text { MULTIPLIER } \\
\text { WALLACE } \\
\text { TREE }\end{array}$ \\
\hline Slice LUTs & 342 & 699 \\
\hline Slice Flip Flops & 74 & 361 \\
\hline Delay & $16.354 \mathrm{~ns}$ & $14.668 \mathrm{~ns}$ \\
\hline Frequency & $61.147 \mathrm{MHz}$ & $68.176 \mathrm{MHz}$ \\
\hline
\end{tabular}

Table 2 Comparison of the Filter with and Without Pipelining

Reference Table 2, 3 Fir - Booth Multiplier with Wallace Tree Proposed Fir -Pipelined -Booth Multiplier Wallace Tree Is Given, The parameters Of Proposed System better Result for Comparing to other existing Result.

\begin{tabular}{|c|c|c|}
\hline $\begin{array}{c}\text { PARA } \\
\text { METE } \\
\text { R }\end{array}$ & $\begin{array}{c}\text { ELECTROC } \\
\text { ARDIOGRA } \\
\text { M - FIR - } \\
\text { BOOTH } \\
\text { MULTIPLIE } \\
\text { R WITH } \\
\text { WALLACE } \\
\text { TREE }\end{array}$ & $\begin{array}{c}\text { ELECTROCAR } \\
\text { DIOGRAM - FIR } \\
\text {-PIPELINED - } \\
\text { BOOTH } \\
\text { MULTIPLIER } \\
\text { WALLACE } \\
\text { TREE }\end{array}$ \\
\hline $\begin{array}{c}\text { Slice } \\
\text { LUTs }\end{array}$ & 1253 & 1997 \\
\hline $\begin{array}{c}\text { Slice } \\
\text { Flip } \\
\text { Flops }\end{array}$ & 299 & 519 \\
\hline $\begin{array}{c}\text { Delay } \\
\text { Frequen } \\
\text { cy }\end{array}$ & $19.383 \mathrm{~ns}$ & $17.128 \mathrm{~ns}$ \\
\hline
\end{tabular}

Table 3 Comparison of Electrocardiogram Signal with and Without Pipelining of the Filter

\section{CONCLUSIONS}

$\mathrm{R}$ peak is determined by using the PAN TOMPKINS algorithm and with the help of this result average mean value is calculated for the detected peak value by the NBC. The average mean value is compared with the stored dataset. The value closer to the data set is taken and normal and abnormal signal is given as output. By comparing the results of the non pipelined filter and the pipelined filter, the non pipelined filter structure has 19.383 ns delay while the pipelined filter structure has 17.128 ns delay. This shows that the pipelined filter structure has less delay of $2.255 \mathrm{~ns}$ than the pipelined filter structure. And the frequency of the non pipelined filter structure is $51.592 \mathrm{MHz}$ and the frequency of the pipelined filter structure is $58.384 \mathrm{MHz}$. This shows that the increase in frequency and speed. This is very useful for cardiologists to find the heart diseases with less delay and high speed.

\section{REFERENCES}

1) R. S. Khandpur, Handbook of biomedical instrumentation: Tata McGraw-Hill Education, 1992.

2) R. M. Rangayyan, Biomedical signal analysis vol. 33: John Wiley \& Sons, 2015.

3) F. Buendía-Fuentes, M. Arnau-Vives, A. ArnauVives, Y. Jiménez- Jiménez, J. Rueda-Soriano, E. Zorio-Grima, et al., "High-bandpass filters in electrocardiography: source of error in the interpretation of the ST segment," ISRN cardiology, vol. 2012, 2012.

4) S. L. Joshi, R. A. Vatti, and R. V. Tornekar, "A survey on ECG signal denoising techniques," in Communication Systems and Network Technologies (CSNT), 2013 International Conference on, 2013, pp. 60- 64.

5) J. S. Sørensen, L. Johannesen, U. S. L. Grove, K. Lundhus, J.-P. Couderc, and C. Graff, "A comparison of IIR and wavelet filtering for noise reduction of the ECG," in Computing in Cardiology, 2010, 2010, pp. 489-492.

6) M. Aqil, A. Jbari, and A. Bourouhou, "Adaptive ECG Wavelet analysis for R-peaks detection," in Electrical and Information Technologies (ICEIT), 2016 International Conference on, 2016, pp. 164167.

7) Safari, H. D. Hesar, M. Mohebbi, and F. Faradji, "A novel method for R-peak detection in noisy ECG signals using EEMD and ICA," in Biomedical Engineering and 2016 1st International Iranian Conference on Biomedical Engineering (ICBME), 2016 23rd Iranian Conference on, 2016, pp. 155-158. 
8) M. Rakshit and S. Das, "An improved EMD based ECG denoising method using adaptive switching mean filter," in Signal Processing and Integrated Networks (SPIN), 2017 4th International Conference on, 2017, pp. 251-255.

9) M. Amiri, M. Afzali, and B. V. Vahdat, "Comparison of different ECG signal power line denoising methods based on SNR improvement," in Biomedical Engineering (ICBME), 2012 19th Iranian Conference of, 2012, pp. 159-162.

10)D. H. Johnson, "Signal-to-noise ratio," Scholarpedia, vol. 1, p. 2088, 2006.

11) Ali, L., Sidek, R. I., Aris, M.A., Ali, M. and Suparjo, B. S.: (Challengesand Directions for IC testing).In: Integration, the VLSI Journal, Elsevier Science, Netherland, 2004, Vol. 37(1), p. 17-28.

12) Obermaisser, R., Kopetz, H. and Paukovits, C.: (A Cross-Domain Multiprocessor System-on-a-Chip for Embedded Real-Time Systems).In: IEEE Transaction on Industrial Informatics, Vol. 6(4), 2010.

13) Song, P., Jiye, H. and Guodong,W.: (Modern DSP Technology [M]).In: Xi'an: Xidian University Press, 2003, China, p. 163-180.

14) R. Acharya, J. S. Suri, J. A.E. Spaan and S.M. Krishnan, Advances in Cardiac Signal Processing, springer, pp. 1-50.

15) W. J. Germann and C. L. Standield, "Principles of Human Physiology," Benjamin Cummings, San rancisco, 2002.

16) J. Moss and S. Stern., "Noninvasive Electro cardiology," Clinical Aspects of Holter, London, Philadelphia, W.B. Saunders, 1996.

17) M. Gabriel Khan, "Rapid ECG interpretation" Third edition, 2003.

18) Ketha, M. B., Venkateswarlu Ch. and Raghuram, K.: (Design \& Fpga Implementation of Reconfigurable Fir Filter Architecture For Dsp Applications). In: International Journal of Engineering Research \& Technology, Vol. I, Issue 7, September 2012.

19) Mirzaei, S., Hosangadi, A. and Kastner, R.: (FPGA Implementation of High Speed FIR Filters Using Add and Shift Method). In: Proceedings of the International Conference of Computer Design, IEEE, 2006.
20) TARUMI, K., HYODO, A. and MUROYAMA, M. and YASUURA, H.: ("A design method for a low power digital FIR Filter indigital wireless communication systems). In: Catalog of Library, System LSI research center, Kyushu Univresity, 2004.

21) J. P. Abenstein and W. J. Tompkins, "A New Data-Reduction Algorithm for Real-Time ECG Analysis," in IEEE Transactions on Biomedical Engineering, vol. BME-29, no. 1, pp. 43-48, Jan. 1982.

22) Tinku Acharya, Ping-Sing Tsai, "JPEG2000 Standard for Image Compression: Concepts, Algorithms and VLSI Architectures", John Wiley \& Sons Inc., Hoboken, New Jersey, 2005.

23) S. Alam, R. Gupta, "A DPCM based ECG coder with thresholding for real time telemonitoring applications", Proc. International Conference on Communication and Signal Processing, Melmaruvathur, 3-5 April 2014, pp. 176-180.

24) D. Gurve, B. S. Saini and I. Saini, "An improved lossless ECG data compression using ASCII character encoding," 2016 International Conference on Wireless Communications, Signal Processing and Networking (WiSPNET), Chennai, India, 2016, pp. 758-764.

25) P. Bera and R. Gupta, "Real-time compression of ECG using dynamic bit allocation strategy," 2016 IEEE First International Conference on Control, Measurement and Instrumentation (CMI), Kolkata, 2016, pp. 21-25.

26) B. S. Pimentel, J.H. de Avila Valgas Filho, R. L. Campos, A. O. Fernandes and C. J. Nunes Coelho, "A FPGA implementation of a DCTbased digital electrocardiographic signal compression device," Integrated Circuits and Systems Design, 2001, 14th Symposium on., Pirenopolis, 2001, pp. 4449 .

27) Y. Yongming, L. Jungang and W. Jianmin, "LADT Arithmetic Improved and Hardware Implemented for FPGA -Based ECG Data Compression," 2007 2nd IEEE Conference on Industrial Electronics and Applications, Harbin, 2007, pp. 2230-2234.

28) S. L. Chen, H. Y. Lee, C. A. Chen, H. Y. Huang and C. H. Luo, "Wireless Body Sensor Network With Adaptive Low-Power Design for Biometrics and Healthcare Applications," in IEEE Systems Journal, vol. 3, no. 4, pp. 398-409, Dec. 2009. 
29) H. Kim, R. F. Yazicioglu, T. Torfs, P. Merken, H. J. Yoo and C. Van Hoof, "A low power ECG signal processor for ambulatory arrhythmia monitoring system," 2010 Symposium on VLSI Circuits, Honolulu, HI, 2010, pp. 19-20.

30) Chua, E., and Fang,W.C.: 'Mixed bio-signal lossless data compressor for portable brain-heart monitoring systems, IEEE Trans. Consum.Electron., 2011, 57, (1), pp. 267-273.

31) S. L. Chen and J. G. Wang, "VLSI implementation of low-power costefficient lossless ECG encoder design for wireless healthcare monitoring application," in Electronics Letters, vol. 49, no. 2, pp. 91-93, January 172013.

32) Guei-An Luo, S. L. Chen and Ting-Lan Lin, "VLSI implementation of a lossless ECG encoder design with fuzzy decision and two-stage Huffman coding for wireless body sensor network," Information, Communications and Signal Processing (ICICS) 2013 9th International Conference on, Tainan, 2013, pp. 1-4.

33) C. J. Deepu, X. Zhang, W. S. Liew, D. L. T. Wong and Y. Lian, "An ECG-SoC with $535 \mathrm{nW} /$ channel lossless data compression for wearable sensors," Solid-State Circuits Conference (A-SSCC), 2013 IEEE Asian, Singapore, 2013, pp. 145-148.

34) Li, K., Pan, Y., Chen, F., Cheng, K.-T., and Huan, R.: Real-time lossless ECG compression for lowpower wearable medical devices based on adaptive region prediction, Electron. Lett., 2014, 50, (25), pp. $1904^{*} 1906$

35) S. L. Chen, M. C. Tuan, T. K. Chil and T. L. Lin, "VLSI architecture of lossless ECG compression design based on fuzzy decision and optimization method for wearable devices," in Electronics Letters, vol. 51, no. 18, pp.1409-1411,9 32015.

36) Rik Vullings, Bert de Vries, and Jan W. M. Bergmans, "An Adaptive Kalman Filter for ECG Signal Enhancement", IEEE Transactions on Biomedical Engineering, Vol 58, April 2011

37) M.Ravi Kumar "ECG (ECG) Signal Processing On FPGA for Emerging Healthcare Applications" International Journal of Electronics Signals and Systems (IJESS) ISSN: 2231- 5969, Vol-1 Iss-3, 2012

38) Mbachu C.B. and Offor K.J. "Reduction of powerline noise in ECG signal using fir digital filter implemented with hamming window" International Journal of Science, Environment and Technology, Vol. 2, No 6, 2013, 1380 - 1387 ISSN 2278-3687 (O).

39) Bhumika Chandrakar1, O.P.Yadav2, V.K.Chandra3 "A survey of noise removal techniques for ECG signals" International Journal of Advanced Research in Computer and Communication Engineering Vol. 2, Issue 3, March 2013 ISSN (Print) : 2319-5940 ISSN (Online) : 2278-1021.

40) M. Yazhini and R. Ramesh, “ FIR Filter Implementation using Modified Distributed Arithmetic Architecture " Indian Journal of Science and Technology | Print ISSN: 0974-6846, Online ISSN: 0974-5645, Vol 6 (5) May 2013

41) R. M. Narsale, Dhanashri Gawali and Amit Kulkarni " FPGA Based Design \& Implementation Of Low Power FIR Filter For ECG Signal Processing" International Journal of Science, Engineering and Technology Research (IJSETR), Volume 3, Issue 6, June 2014 